\title{
ANALYTICAL DESCRIPTIONS OF A TEMPERATURE FIELD IN A MASSIVE BODY CAUSED BY MOVED VOLUMETRIC HEAT SOURCE MODEL FOR APPLICATION TO WELDING PROCESSES
}

\author{
Jerzy Winczek \\ Institute of Mechanical Technologies, Czestochowa University of Technology \\ Czestochowa, Poland \\ j.winczek@imipkm.pcz.pl
}

\begin{abstract}
In many manufacturing processes (e.g. welding, surfacing, rebuilding, laser heat treatment) a moving heat source (electrode, laser beam) is used. In the work the temperature field descriptions for selected welding processes have been presented. In the solutions, the volumetric heat source models with axis perpendicular and tilted towards the direction of motion have been applied. A theoretical consideration has been illustrated by results of numerical simulations of the temperature field during multi-pass weave bead building up and single-pass weld surfacing. Suggested models enable the analysis of the temperature field in a whole body and welding heat cycles at any place of a heated element. The solution determines an origin for a further thermo-mechanical state analysis: phase transformations and stresses.
\end{abstract}

Keywords: temperature field, welding, surfacing, modelling

\section{Introduction}

\subsection{General classification of heated body models and heat sources models}

In order to enable mathematical description of complex phenomena related to heat flow modeling in welding processes, simplified models of bodies and heat source types are defined.

The basic models of heated bodies are (Fig. 1) [1]:

- infinite body - a body infinitely extended in directions $O x, O y, O z$ (Fig. 1a),

- massive body (semi-infinite) - infinitely extended only in directions $O x$ and $O y$. The heat source occurs on a surface with a coordinate $z=0$ (Fig. 1b). This model corresponds to a plate with a thickness of more than about $25 \mathrm{~mm}$,

- plate - body bounded by two planes $z=0$ and $z=\mathrm{g}$, infinite in directions $\mathrm{O} x$ and $\mathrm{O} y$ (Fig. 1c). Thickness $g$ limits and distorts free heat flow in the direction $\mathrm{O} z$. This model corresponds to a plate with the thickness of about $3<g<25 \mathrm{~mm}$,

- thin plate - a model similar to a plate, but with such a small thickness in relation to the source power, that the heat flow isotherms are perpendicular to the plane of the coating. The coating model corresponds to a plate with the thickness $g<3 \mathrm{~mm}$,

- rod - a body infinitely extended in direction $O x$ (Fig. 1e). 


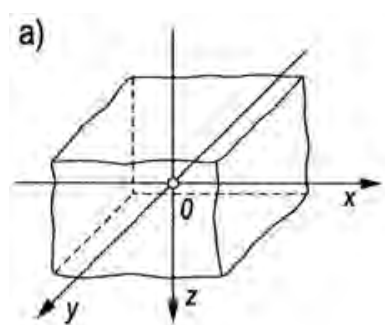

d)

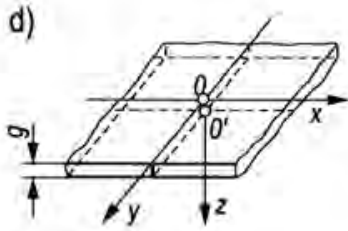

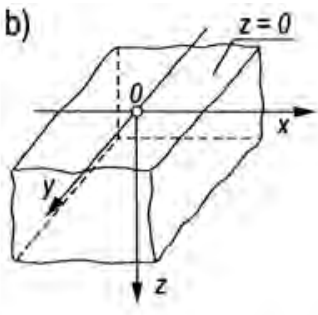
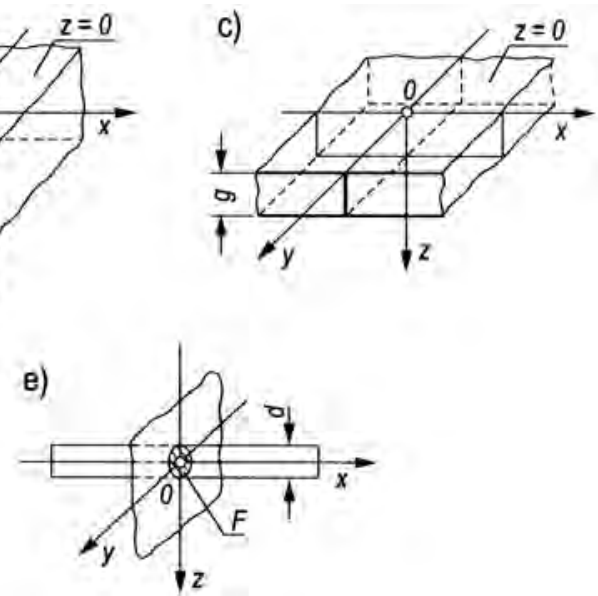

Fig. 1. Basic models of heated bodies: a) infinite body, b) semi-infinite body (massive), c) plate, d) thin plate, e) rod [1]

Due to the shape, the sources acting on the heated body are divided into:

- point - with infinitely small volume,

- linear - evenly distributed in the volume of a cylinder of a small diameter, penetrating through the entire thickness of the body, e.g. a thin coating,

- flat - evenly distributed on the surface of the heated body, e.g. during the butt friction welding of the rod,

- surficial - acting on surfaces with a variable power density, e.g. with Gaussian distribution,

- volumetric - three-dimensional.

In terms of movement speed we distinguish fixed, mobile and mobile high-speed heat sources (at a speed close to the heat flow rate in the material). And in terms of action time, we distinguish a continuous heat source (with constant thermal power) and an instantaneous heat source whose operation time tends to zero.

\subsection{Analytical solutions of the heat conduction equation for point source}

First attempts to describe the temperature field in the welding process and related processes were undertaken in the middle of the last century. Rosenthal [2] and Rykalin [3] works initiated modeling of the temperature field caused by a moving source. Rosenthal was the first to obtain the equation of the temperature distribution for a quasi-steady state for different heat source models (point, linear and surface) by introducing temperature-independent thermo-mechanical properties of the heated material. Rosenthal adopted a heat source in the form of a segment parallel to the axis of the weld, and Rykalin in the form of a perpendicular segment.

For an analytical description of the temperature field, the solution of the differential heat conduction equation is commonly used [4]: 


$$
a \nabla^{2} T(\mathbf{r}, t)=\frac{\partial T(\mathbf{r}, t)}{\partial t}-\frac{Q}{c \rho}
$$

where $\mathbf{r}$ is the radius vector of the considered area point with respect to the heat source, $T$ - temperature, $t$ - time, $a$ - thermal diffusivity, $c$ - specific heat, $\rho$ - density, $Q$ - heat source.

In the case of a point source for an infinite body, the equation solution (1) becomes:

$$
T(R, t)-T_{0}=\frac{Q}{c \rho(4 \pi a t)^{1.5}} \exp \left(-R^{2} / 4 a t\right)
$$

where $R$ is the distance of the considered point from the source, $T_{0}$ - initial temperature of body. For a semi-infinite body the equation solution has the form:

$$
T(R, t)-T_{0}=\frac{2 Q}{c \rho(4 \pi a t)^{1.5}} \exp \left(-R^{2} / 4 a t\right)
$$

or

$$
T(x, y, z, t)-T_{0}=\frac{2 Q}{c \rho(4 \pi a t)^{1.5}} \exp \left(-\frac{\left(x-x^{\prime}\right)^{2}+\left(y-y^{\prime}\right)^{2}+z^{2}}{4 a t}\right)
$$

Equation (4) determines the temperature value at the point with coordinates $x, y$, $z$ with the source position at $x^{\prime}, y^{\prime}$.

Figure 2 shows a temperature distribution in the semi-infinite body caused by the instantaneous point heat source (4). Thermal properties of the material were assumed in the calculations $a=8 \cdot 10^{-6} \mathrm{~m}^{2} / \mathrm{s}, c \rho=5,2 \cdot 10^{6} \mathrm{~J} / \mathrm{m}^{\circ} \mathrm{C}, Q=3,3 \cdot 10^{3} \mathrm{~J}$ and $T_{0}=0$.
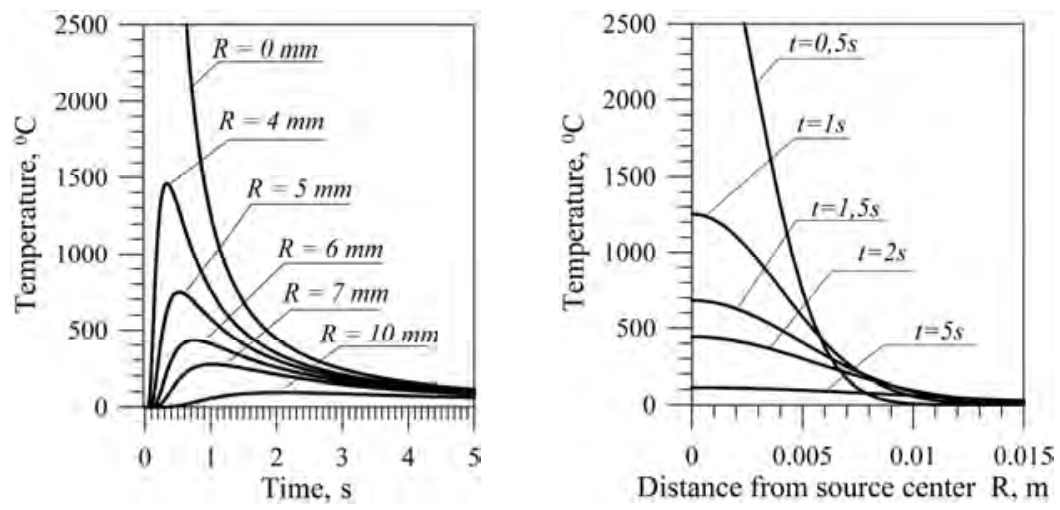

Fig. 2. Temperature near the temporary heat source: a) heat cycles for points at different distances from the source; b) temperature distribution in the body for selected times 
In the case of a point source on the surface of the semi-infinite body, moving along the $x$ axis of the Cartesian system from $x_{0}$ point at speed $v$, the temperature field at time $t$ from the beginning of action of the welding heat source at the point with coordinates $x, y, z$ (Fig. 3) will be expressed by the sum of the temperature rises caused by the heat release at elementary time increments $d t$ ':

$$
T(x, y, z, t)-T_{0}=\int_{0}^{t} \frac{2 \dot{q}}{c \rho\left[4 \pi a\left(t-t^{\prime}\right)\right]^{1.5}} \exp \left(-\frac{\left(x-v t^{\prime}-x_{0}\right)^{2}+y^{2}+z^{2}}{4 a\left(t-t^{\prime}\right)}\right) d t^{\prime}
$$

where $x_{0}$ is the coordinate of beginning of the source path and $\dot{q}$ its power.

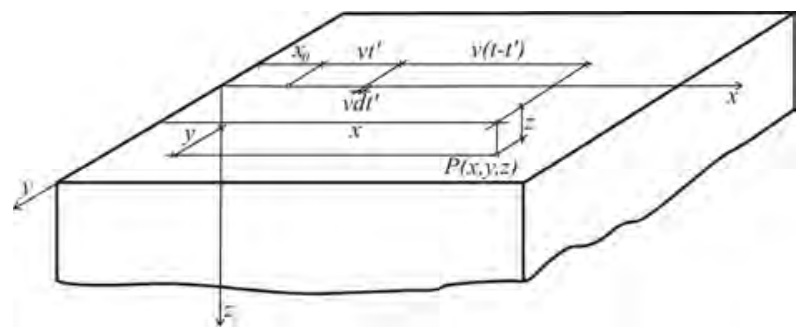

Fig. 3. Scheme for determining of the temporary temperature during heating of moving heat source

Since then, many researchers have tried to obtain a solution closest to the real temperature distribution.

\section{Surface and volumetric heat source models}

Eagar and Tsai [5] modified Rosenthal's model by introducing a surface heat source (6) with Gaussian distribution (Fig. 4) to the description of the temperature field in the semi-infinite body. Gaussian distribution is characterized by averaged radius $r_{0}$ in which the heat volume $q_{\max } / e$.

$$
q(x, y)=\frac{q_{\max }}{2 \pi\left(r_{0}\right)^{2}} \exp \left(-\left(x^{2}+y^{2}\right) / 2 r_{0}^{2}\right)
$$

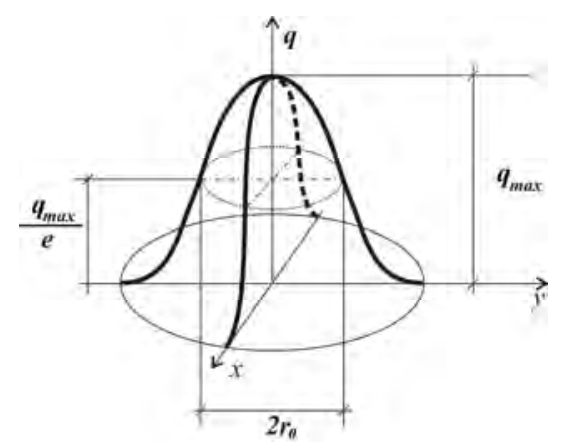

Fig. 4. Surface two-dimensional Gaussian distributed heat source 
A double-ellipsoidal, three-dimensional heat source (Fig. 5) was first introduced by Goldak [6]. Two different semi-ellipses were combined to give a new heat source. The heat flux for each of the ellipses is described by a different equation. For the points $(x, y, z)$ belonging to a semi-ellipse located in the front part of the welding arc, the heat flux equation is presented as:

$$
Q(x, y, z)=\frac{6 \sqrt{3} r_{f} Q}{a_{h} b_{h} c_{h f} \pi \sqrt{\pi}} \exp \left(-\frac{3 x^{2}}{c_{h f}^{2}}-\frac{3 y^{2}}{a_{h}^{2}}-\frac{3 z^{2}}{b_{h}^{2}}\right)
$$

and for the points $(x, y, z)$ belonging to a semi-ellipse coinciding with the rear part of the arc:

$$
Q(x, y, z)=\frac{6 \sqrt{3} r_{b} Q}{a_{h} b_{h} c_{h b} \pi \sqrt{\pi}} \exp \left(-\frac{3 x^{2}}{c_{h b}^{2}}-\frac{3 y^{2}}{a_{h}^{2}}-\frac{3 z^{2}}{b_{h}^{2}}\right)
$$

where $a_{h}, b_{h}, c_{h f}, c_{h b}$ are the parameters of the ellipsoidal heat source, $Q$ the applied arc heat, and $r_{f}, r_{b}$ proportionality coefficients corresponding to the heat distribution in the front and rear parts of the heat source, where $r_{f}+r_{b}=2$.

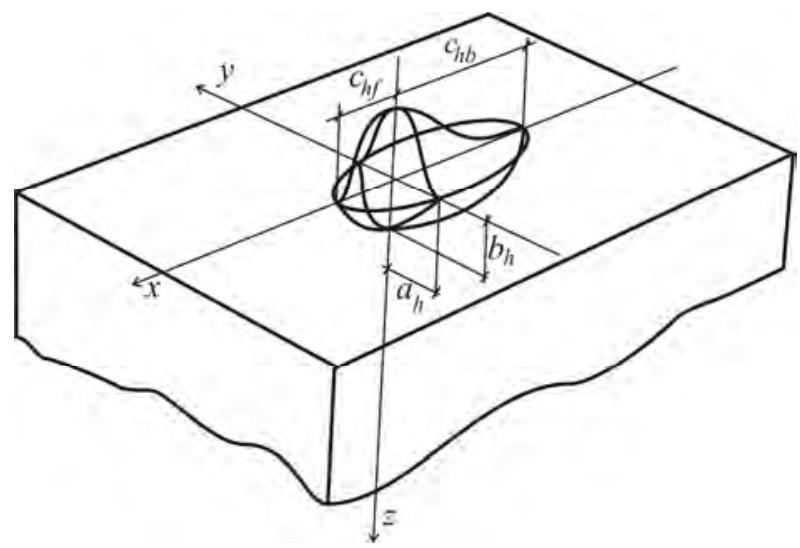

Fig. 5. Double ellipsoidal Goldak's model of heat source

Hongyuan et al. [7] proposed a tilted double ellipsoidal heat source (Fig. 6) (9)-(10) to calculate the temperature field using the finite element method. Importing the energy fraction $f_{f}+f_{r}=2$ gives the heat source formula for a forward half ellipsoid:

$$
\begin{gathered}
Q(x, y, z, t)=Q(0) f_{f} \exp \left\{-3[x+v(\tau-t)]^{2} / \frac{a^{2}}{\sin \beta}\right\} \\
\exp \left(-3 y^{2} / \frac{b^{2}}{\sin \gamma}\right) \exp \left[-3 z^{2} /(c \cos \theta)^{2}\right]
\end{gathered}
$$


and for a rear half of ellipsoid:

$$
\begin{gathered}
Q(x, y, z, t)=Q(0) f_{r} \exp \left\{-3[x+v(\tau-t)]^{2} / \frac{a^{2}}{\sin \beta}\right\} \\
\exp \left(-3 y^{2} / \frac{b^{2}}{\sin \gamma}\right) \exp \left[-3 z^{2} /(c \cos \theta)^{2}\right]
\end{gathered}
$$

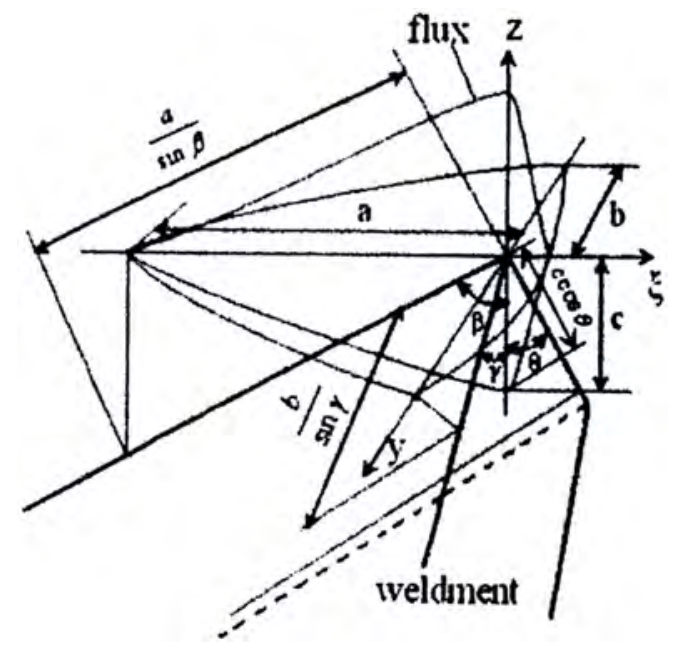

Fig. 6. Double ellipsoidal Goldak's tilted model of heat source [7]

In [8] description of three dimensional temperature field a volumetric heat source of Gaussian surface distribution is assumed (Fig. 7) with parabolic change in relation to depth (Fig. 8).

$$
q_{v}=q_{\max } \exp \left(-\frac{\left(x^{\prime}\right)^{2}+\left(x^{\prime}\right)^{2}}{r_{B}^{2}}\right)\left(1-\left(\frac{z^{\prime}}{z_{0}}\right)^{2}\right)
$$

where $z_{0}$ depth of heat source deposition, and $r_{B}$ averaging radius of distribution of the Gaussian heat source.

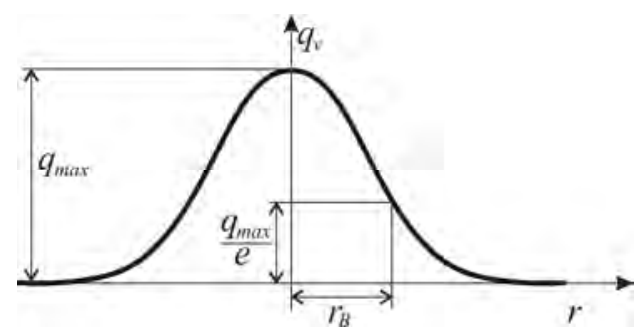

Fig. 7. Gaussian surface distribution of the heat source 


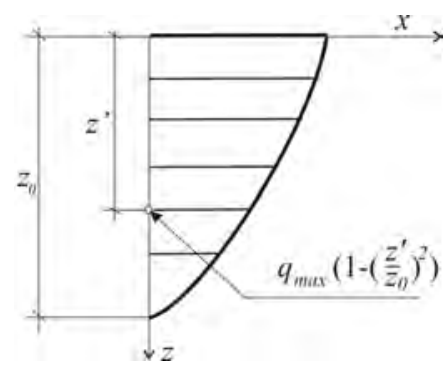

Fig. 8. Parabolic change of the heat source in relation to depth

The analytical solution of a temporary temperature field in half-infinite body caused by moving heat source tilted towards the direction of motion (Fig. 9) is presented in [9] (12)-(14).

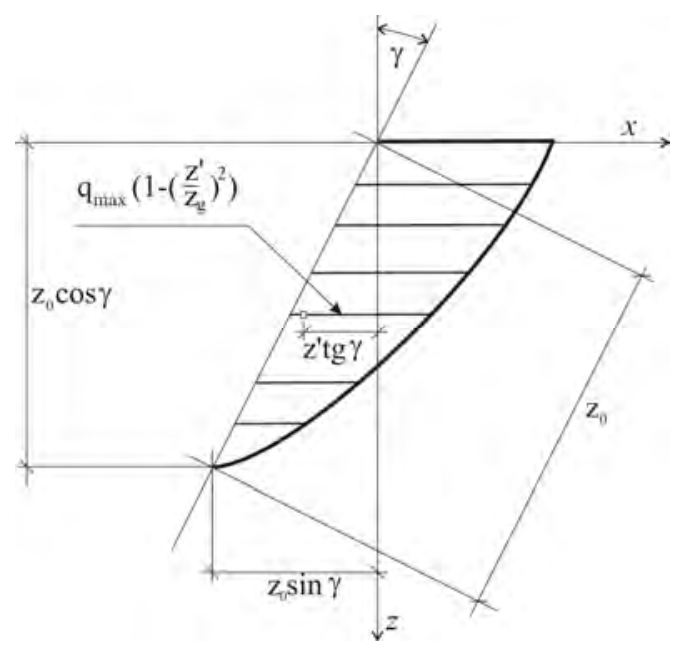

Fig. 9. Distribution of tilted heat source in relation to depth

$$
q_{v}=q_{\max } \exp \left(-\frac{\left(x^{\prime}+z^{\prime} \operatorname{tg} \gamma\right)^{2}+\left(y^{\prime}\right)^{2}}{r_{B}^{2}}\right)\left(1-\left(\frac{z^{\prime}}{z_{g}}\right)^{2}\right)
$$

for $z^{\prime} \in\left\langle 0, z_{g}\right\rangle$ and $-\pi / 2<\gamma<\pi / 2$, which fulfills the condition

$$
q=\int_{-\infty}^{\infty}\left(\int_{-\infty}^{\infty}\left(\int_{0}^{z_{g}} q_{v} d z^{\prime}\right) d x^{\prime}\right) d y^{\prime}
$$

where $z_{g}$ denotes

$$
z_{g}=z_{0} \cos \gamma
$$




\section{The temperature field descriptions for selected welding processes}

\subsection{Modelling of the temperature field in a rectangular steel plate heated by weave moving heat source}

In solution, the zig-zag trajectory of heat source has been accepted, simulated commonly used in practice a weaving motion of a welding electrode. It comes into existence as a result of association heat source movement along a heated plate with synchronous swings across the endwise movement.

Based on works $[8,10,11]$ for a taken zig-zag trajectory (Fig. 10) by heat source $Q$ with power $q[\mathrm{~J} / \mathrm{s}]$, speed of welding head $v[\mathrm{~m} / \mathrm{s}]$, and speed $w[\mathrm{~m} / \mathrm{s}]$ crosswise motion, temperature field at point with coordinates $(x, y, z) \eta$ meters away from the source along the $y$ axis and $\xi$ along the $x$ axis can be described by
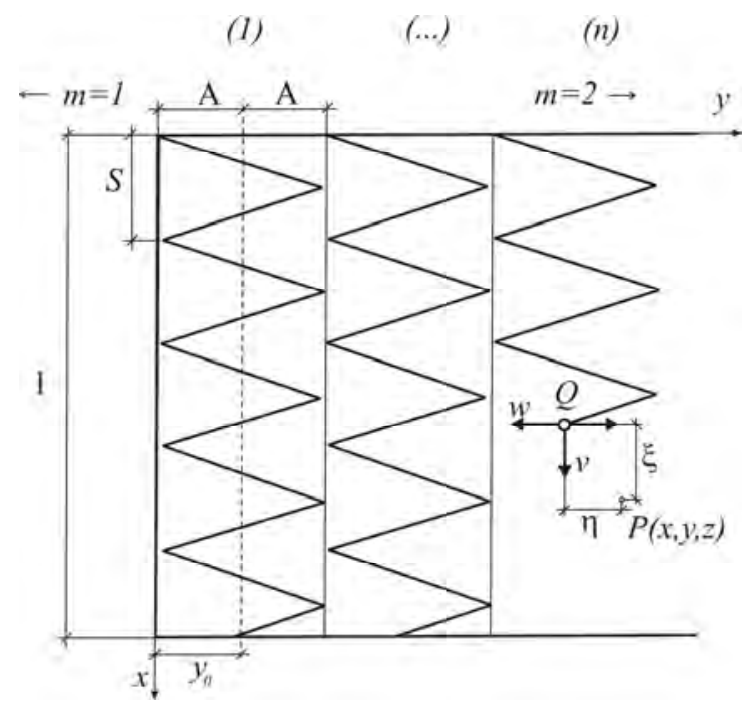

Fig. 10. Schematic diagram of heat source trajectory

- for time $t \leq t_{t}$ where $t_{t}=n_{p}\left(l / v+t_{p}\right)-t_{p}$ means total run time $n_{p}$ endwise movements:

$$
T\left(x, y, z, z_{0}, t\right)-T_{0}=\sum_{j=1}^{j=k-1} A \int_{(j-1) t_{p}}^{j t_{p}} F_{C}\left(t^{\prime}\right) d t^{\prime}+A_{k} \int_{j t_{p}}^{t} F_{H}\left(t^{\prime \prime}\right) d t^{\prime \prime}
$$

- for time $t>t_{t}$ :

$$
T\left(x, y, z, z_{0}, t\right)-T_{0}=\sum_{j=1}^{k c} A \int_{(j-1) t_{p}}^{j t_{p}} F_{C}\left(t^{\prime}\right) d t^{\prime}
$$


where:

$$
\begin{aligned}
& A=\frac{3}{8} \frac{q}{c \rho \pi a z_{0}} \\
& A_{k}=\frac{3}{8} \frac{q}{c \rho \pi a z_{0}} \exp \left(-\frac{\xi v-\eta_{k}(-1)^{k} w}{2 a}-\frac{\left(v^{2}+w^{2}\right) t_{0}}{4 a}\right) \\
& F_{C}\left(t^{\prime}\right)=\frac{1}{t+t_{0}-t^{\prime}} \exp \left(-\frac{\left(x-v t^{\prime}\right)^{2}}{4 a\left(t+t_{0}-t^{\prime}\right)}+\right. \\
& -\frac{\left(y-(-1)^{k} A+(-1)^{k} w\left(t^{\prime}-(k-1) t_{p}\right)-y_{0}-(-1)^{m} 2(n-1) A\right)^{2}}{4 a\left(t+t_{0}-t^{\prime}\right)} \\
& \left(( 1 - \frac { z ^ { 2 } + 2 a ( t - t ^ { \prime } ) } { z _ { 0 } ^ { 2 } } ) \left(\operatorname { e r f } \left(\frac{z+z_{0}}{2\left(a\left(t-t^{\prime}\right)\right)^{0.5}}+\right.\right.\right. \\
& \left.-\Phi(z) \operatorname{erf}\left(\Phi(z) \frac{z-z_{0}}{2\left(a\left(t-t^{\prime}\right)\right)^{0.5}}\right)\right)+ \\
& \frac{4 a\left(t-t^{\prime}\right)}{z_{0}^{2}}\left(\frac{z+z_{0}}{\left(4 \pi a\left(t-t^{\prime}\right)\right)^{0.5}} \exp \left(-\frac{\left(z-z_{0}\right)^{2}}{4 a\left(t-t^{\prime}\right)}\right)+\right. \\
& \left.\left.\left.-\frac{z-z_{0}}{\left.\left(4 \pi a\left(t-t^{\prime}\right)\right)\right)^{0.5}} \exp \left(-\frac{\left(z+z_{0}\right)^{2}}{4 a\left(t-t^{\prime}\right)}\right)\right)\right)\right) \\
& F_{H}\left(t^{\prime \prime}\right)=\frac{1}{t_{0}+t^{\prime \prime}} \exp \left(-\frac{\xi^{2}+\eta^{2}}{4 a\left(t_{0}+t^{\prime \prime}\right)}-\frac{v^{2} t^{\prime \prime}}{4 a}\right) \\
& \left(\left(1-\frac{z^{2}+2 a t^{\prime \prime}}{z_{0}^{2}}\right)\left(\operatorname{erf}\left(\frac{z+z_{0}}{2\left(a t^{\prime \prime}\right)^{1 / 2}}\right)-\Phi(z) \operatorname{erf}\left(\Phi(z) \frac{z-z_{0}}{2\left(a t^{\prime \prime}\right)^{1 / 2}}\right)\right)+\right. \\
& \frac{4 a t^{\prime \prime}}{z_{0}^{2}}\left(\frac{z+z_{0}}{\left(4 \pi a t^{\prime \prime}\right)^{1 / 2}} \exp \left(-\frac{\left(z-z_{0}\right)^{2}}{4 a t^{\prime \prime}}\right)-\frac{z-z_{0}}{\left(4 \pi a t^{\prime \prime}\right)^{1 / 2}} \exp \left(-\frac{\left(z+z_{0}\right)^{2}}{4 a t^{\prime \prime}}\right)\right) \\
& \eta=y-(-1)^{k} A+(-1)^{k} w\left(t-(k-1) t_{p}-t_{0}\right)-y_{0}-(-1)^{m} 2(n-1) A \\
& \xi=x-v\left(t+t_{0}\right)
\end{aligned}
$$




$$
\begin{gathered}
t_{p}=2 A / w \\
\xi=x-v\left(t+t_{0}\right)
\end{gathered}
$$

$z_{0}$ - the depth of heat source deposition, $t^{\prime}$ - time, that passed since the start of the heat source [s], S - weld bead pitch, $y_{0}$ - coordinate y location of the axis of the first padding weld, $n$ - number of the current weld pad, $m$ - the order of the overlaying next padding welds coefficient, for $m=2$ direction of the order of overlaying next padding welds is compatible with the direction of the axis $y$, for $m=1$ direction is opposite, $A$ - amplitude of weld bead, distance between opposite vertexes of the weld bead amounts $2 A, t_{0}$ characterize superficial distribution of the heat source, whereas $r_{B}^{2}=4 a t_{0}$ [10]. In calculations of the temperature field while making $n$ runs, temperature-rises with the next transition of the welding heat source and self-cooling of already overlaid padding welds as well as already heated areas, is defined by the equation:

$$
T(x, y, z, t)=\sum_{k=1}^{n} T_{i}(x, y, z, t)
$$

are taken into consideration.

\subsubsection{Example of calculations}

Calculations of the temperature field have been carried out while rebuilding the surface of a rectangular prismatic steel element. Overall dimensions of the detail: $500 \times 160 \times 50 \mathrm{~mm}$. In calculations, source power is $2700 \mathrm{~W}$ in Gaussian distribution of the density power defined by $t_{0}=1 \mathrm{~s}$ and the depth of heat source deposition $z_{0}=0.006 \mathrm{~m}$. Power of the heat source corresponds to rebuilding with $150 \mathrm{~A}$ current, $24 \mathrm{~V}$ voltage and arc efficiency $\eta_{e}=0.75$. Thermal properties have been defined by thermal diffusivity $a=8.012 \cdot 10^{-6} \mathrm{~m}^{2} / \mathrm{s}$, and thermal capacity $c \rho=5225126.4 \mathrm{~J} / \mathrm{Km}^{3}$. Technological parameters of welding head motion defined by the speed along the weld bead $v=0.00083 \mathrm{~m} / \mathrm{s}$, frequency of crosswise swings $f=0.21 / \mathrm{s}$ and amplitude $A=0,01 \mathrm{~m}$, give the weld bead pitch $S=0.004 \mathrm{~m}$ and the speed of crosswise electrode motion $w=0.008 \mathrm{~m} / \mathrm{s}$. The area of decrements $0.08 \mathrm{~m}$ wide and $0.002 \mathrm{~m}$ deep in the middle part of the cast has been padded along $l=0.2 \mathrm{~m}$ by four weld beads, whereas the order of making padding welds was compatible with direction of $y$ axes. The coordinate centre line of a first weld pass is $y_{0}=0.05 \mathrm{~m}$. The geometry of regenerated area in cross section of cast steel is illustrated in Figure 11.

Figure 12 presents the trajectory of a welding electrode during the whole welding process. After a particular each weld pass occurs technological pause in action of the heat source $\left(t_{p}=2 \mathrm{~s}\right)$ for transition to the begin of the next weld bead. 


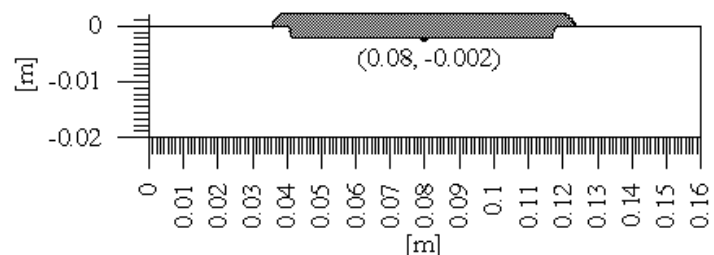

Fig. 11. Schematic diagram of heat source trajectory

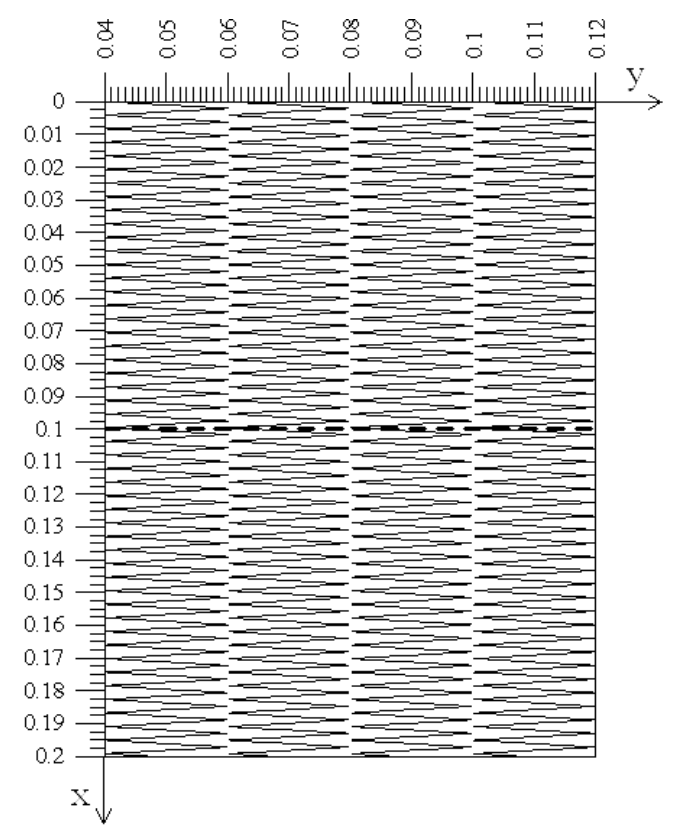

Fig. 12. Schematic diagram of heat source trajectory

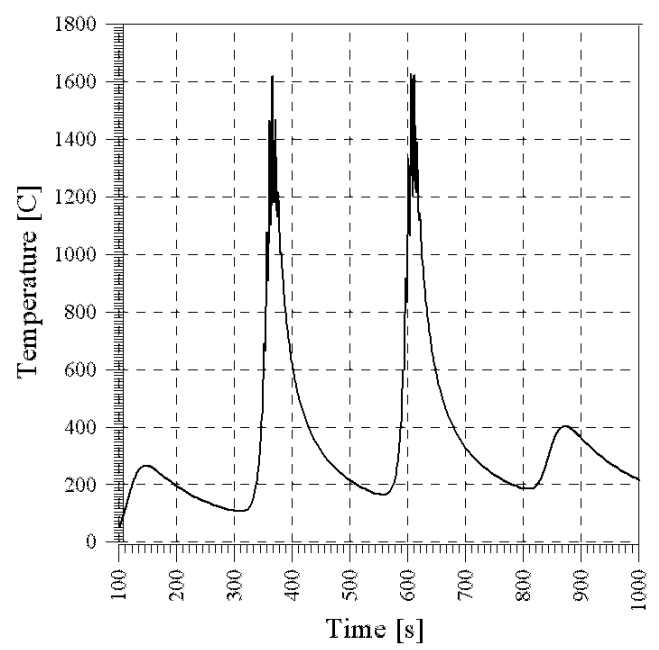

Fig. 13. Welding thermal cycles at point $(0.08,-0.002)$ 


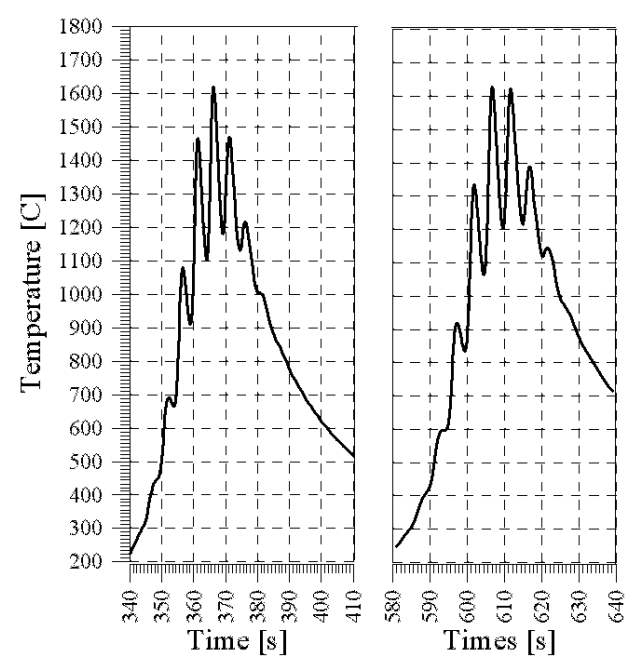

Fig. 14. Selected fragments of welding thermal cycles at point $(0.08,-0.002)$

The solution presented by equations (15)-(25) enables one to calculate the temperature field at any place and time of a welding object. In Figure 13, the welding thermal cycle at point $(0.08,-0.002)$ - see Figure 11 , at a point of contact of the second and the third pads, is presented. For separate welds, the maximum peak temperatures of $265,1619,1629$ and $402^{\circ} \mathrm{C}$ were calculated. However during overlaying, the second and the third weld bead comes to multiple heating and self-cooling, including triple weld penetration as observed in Figure 14.

\subsection{The model of the temperature field taking into account a tilt of heat source}

In welding processes, the tilt of the electrode toward to the movement direction is often used. In addition to the perpendicular electrode to the surface, two techniques are used (Fig. 15): a) with the electrode inclination toward the direction of motion; b) with the electrode inclined in the direction opposite to its movement.

a)

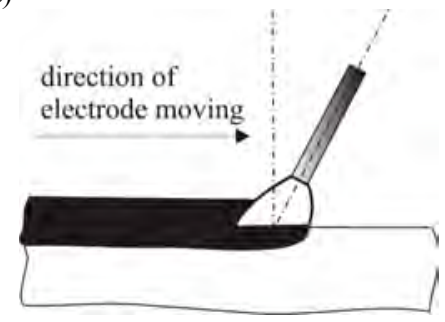

b)

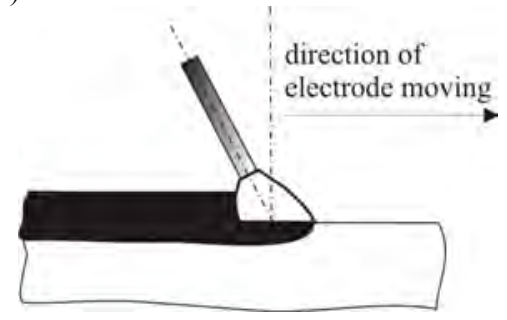

Fig. 15. Scheme of weld surfacing with tilted electrode: a) electrode inclined in the direction opposite to its movement, b) electrode inclination toward direction of motion 
Taking into consideration the motion of heat source (10)-(12) with travel speed $v$ and coordinates $\left(x_{0}, y_{0}\right)$ of initial position of heat source, the temperature field is described by equations (Fig. 16):

- for time $t \leq t_{c}$, where $t_{c}$ means the total time of weld application:

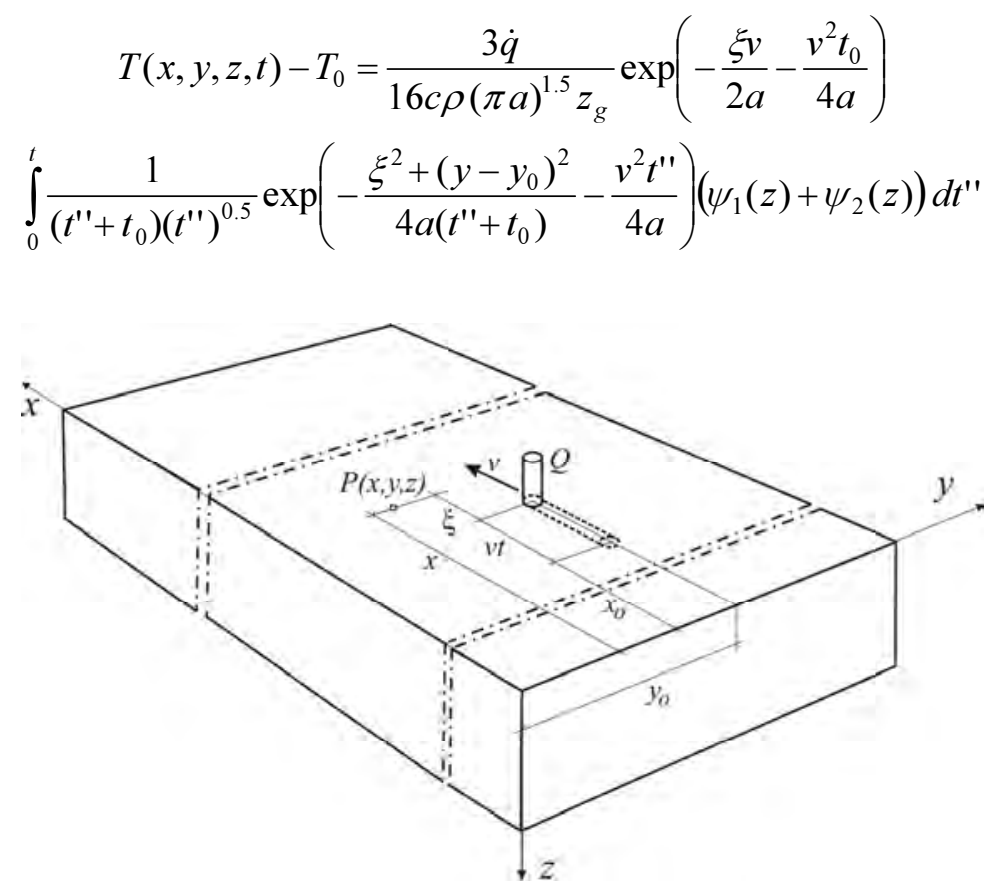

Fig. 16. Scheme for determining temporary distance between selected point of object and heat source

- for time $t \leq t_{c}$, where $t_{c}$ means the total time of weld application:

$$
\begin{gathered}
T(x, y, z, t)-T_{0}=\frac{3 \dot{q}}{16 c \rho(\pi a)^{1.5} z_{g}} \exp \left(-\frac{\xi v}{2 a}-\frac{v^{2} t_{0}}{4 a}\right) \\
\int_{0}^{t} \frac{1}{\left(t^{\prime \prime}+t_{0}\right)\left(t^{\prime \prime}\right)^{0.5}} \exp \left(-\frac{\xi^{2}+\left(y-y_{0}\right)^{2}}{4 a\left(t^{\prime \prime}+t_{0}\right)}-\frac{v^{2} t^{\prime \prime}}{4 a}\right)\left(\psi_{1}(z)+\psi_{2}(z)\right) d t^{\prime \prime}
\end{gathered}
$$

- for time $t>t_{c}$ :

$$
\begin{gathered}
T(x, y, z, t)-T_{0}=\frac{3 \dot{q}}{16 c \rho(\pi a)^{1.5} z_{g}} \\
\int_{0}^{t} \frac{1}{\left(t+t_{0}-t^{\prime}\right)\left(t-t^{\prime}\right)^{0.5}} \exp \left(-\frac{\left(x-v t^{\prime}-x_{0}\right)^{2}+\left(y-y_{0}\right)^{2}}{4 a\left(t+t_{0}-t^{\prime}\right)}\right)\left(\psi_{3}(z)+\psi_{4}(z)\right) d t^{\prime}
\end{gathered}
$$


where:

$$
\begin{aligned}
& \psi_{1}(z)=\frac{D_{H}^{0.5}}{8 A_{H}^{2.5} z_{g}^{2}} \exp \left(-\frac{A_{H} z_{g}^{2}+B_{H} z_{g}+C_{H}}{D_{H}}\right) \\
& \left\{2 A_{H}^{0.5} D_{H}^{0.5}\left[B_{H}\left(-1+\exp \left(\frac{A_{H} z_{g}^{2}+B_{H} z_{g}}{D_{H}}\right)\right)+2 A_{H} z_{g}\right]+\right. \\
& +\exp \left(\frac{\left(2 A_{H} z_{g}+B_{H}\right)^{2}}{4 A_{H} D_{H}}\right) \pi^{0.5}\left[-4 A_{H}^{2} z_{g}^{2}+B_{H}^{2}+2 A_{H} D_{H}\right] \\
& \left.\left[\operatorname{erf}\left(\frac{B_{H}}{2 A_{H}^{0.5} D_{H}^{0.5}}\right)-\operatorname{erf}\left(\frac{2 A_{H} z_{g}+B_{H}}{2 A_{H}^{0.5} D_{H}^{0.5}}\right)\right]\right\} \\
& \psi_{2}(z)=\frac{D_{H}^{0.5}}{8 A_{H}^{2.5} z_{g}^{2}} \exp \left(-\frac{A_{H} z_{g}^{2}+E_{H} z_{g}+C_{H}}{D_{H}}\right) \\
& \left\{2 A_{H}^{0.5} D_{H}^{0.5}\left[E_{H}\left(-1+\exp \left(\frac{A_{H} z_{g}^{2}+E_{H} z_{g}}{D_{H}}\right)\right)+2 A_{H} z_{g}\right]+\right. \\
& +\exp \left(\frac{\left(2 A_{H} z_{g}+E_{H}\right)^{2}}{4 A_{H} D_{H}}\right) \pi^{0.5}\left[-4 A_{H}^{2} z_{g}^{2}+E_{H}^{2}+2 A_{H} D_{H}\right] \\
& \left.\left[\operatorname{erf}\left(\frac{E_{H}}{2 A_{H}^{0.5} D_{H}^{0.5}}\right)-\operatorname{erf}\left(\frac{2 A_{H} z_{g}+E_{H}}{2 A_{H}^{0.5} D_{H}^{0.5}}\right)\right]\right\} \\
& \psi_{3}(z)=\frac{D_{C}^{0.5}}{8 A_{C}^{2.5} z_{g}^{2}} \exp \left(-\frac{A_{C} z_{g}^{2}+B_{C} z_{g}+C_{C}}{D_{C}}\right) \\
& \left\{2 A_{C}^{0.5} D_{C}^{0.5}\left[B_{C}\left(-1+\exp \left(\frac{A_{C} z_{g}^{2}+B_{C} z_{g}}{D_{C}}\right)\right)+2 A_{C} z_{g}\right]+\right. \\
& +\exp \left(\frac{\left(2 A_{C} z_{g}+B_{C}\right)^{2}}{4 A_{C} D_{C}}\right) \pi^{0.5}\left[-4 A_{C}^{2} z_{g}^{2}+B_{C}^{2}+2 A_{C} D_{C}\right] \\
& \left.\left[\operatorname{erf}\left(\frac{B_{C}}{2 A_{C}^{0.5} D_{C}^{0.5}}\right)-\operatorname{erf}\left(\frac{2 A_{C} z_{g}+B_{C}}{2 A_{C}^{0.5} D_{C}^{0.5}}\right)\right]\right\}
\end{aligned}
$$




$$
\begin{aligned}
& \psi_{4}(z)=\frac{D_{C}^{0.5}}{8 A_{C}^{2.5} z_{g}^{2}} \exp \left(-\frac{A_{C} z_{g}^{2}+E_{C} z_{g}+C_{C}}{D_{C}}\right) \\
& \left\{2 A_{C}^{0.5} D_{C}^{0.5}\left[E_{C}\left(-1+\exp \left(\frac{A_{C} z_{g}^{2}+E_{C} z_{g}}{D_{C}}\right)\right)+2 A_{C} z_{g}\right]+\right. \\
& +\exp \left(\frac{\left(2 A_{C} z_{g}+E_{C}\right)^{2}}{4 A_{C} D_{C}}\right) \pi^{0.5}\left[-4 A_{C}^{2} z_{g}^{2}+E_{C}^{2}+2 A_{C} D_{C}\right] \\
& \left.\left[\operatorname{erf}\left(\frac{E_{C}}{2 A_{C}^{0.5} D_{C}^{0.5}}\right)-\operatorname{erf}\left(\frac{2 A_{C} z_{g}+E_{C}}{2 A_{C}^{0.5} D_{C}^{0.5}}\right)\right]\right\} \\
& \psi_{4}(z)=\frac{D_{C}^{0.5}}{8 A_{C}^{2.5} z_{g}^{2}} \exp \left(-\frac{A_{C} z_{g}^{2}+E_{C} z_{g}+C_{C}}{D_{C}}\right) \\
& \left\{2 A_{C}^{0.5} D_{C}^{0.5}\left[E_{C}\left(-1+\exp \left(\frac{A_{C} z_{g}^{2}+E_{C} z_{g}}{D_{C}}\right)\right)+2 A_{C} z_{g}\right]+\right. \\
& +\exp \left(\frac{\left(2 A_{C} z_{g}+E_{C}\right)^{2}}{4 A_{C} D_{C}}\right) \pi^{0.5}\left[-4 A_{C}^{2} z_{g}^{2}+E_{C}^{2}+2 A_{C} D_{C}\right] \\
& \left.\left[\operatorname{erf}\left(\frac{E_{C}}{2 A_{C}^{0.5} D_{C}^{0.5}}\right)-\operatorname{erf}\left(\frac{2 A_{C} z_{g}+E_{C}}{2 A_{C}^{0.5} D_{C}^{0.5}}\right)\right]\right\} \\
& A_{H}=t_{0}+t^{\prime \prime}\left(1+\operatorname{tg}^{2} \gamma\right) \\
& B_{H}=2\left(t^{\prime \prime}\left(\xi+v\left(t^{\prime \prime}+t_{0}\right)\right) \operatorname{tg} \gamma-\left(t_{0}+t^{\prime \prime}\right) z\right) \\
& C_{H}=z^{2}\left(t_{0}+t^{\prime \prime}\right) \\
& D_{H}=4 a t^{\prime \prime}\left(t_{0}+t^{\prime \prime}\right) \\
& E_{H}=2\left(t^{\prime \prime}\left(\xi+v\left(t^{\prime \prime}+t_{0}\right)\right) \operatorname{tg} \gamma+\left(t_{0}+t^{\prime \prime}\right)(z)\right. \\
& A_{C}=t_{0}+\left(t-t^{\prime}\right)\left(1+\operatorname{tg}^{2} \gamma\right) \\
& B_{C}=2\left(\left(t-t^{\prime}\right)\left(x-v t^{\prime}-x_{0}\right) \operatorname{tg} \gamma-\left(t_{0}+t-t^{\prime}\right) z\right)
\end{aligned}
$$




$$
\begin{gathered}
C_{C}=z^{2}\left(t_{0}+t-t^{\prime}\right) \\
D_{C}=4 a\left(t-t^{\prime}\right)\left(t_{0}+t-t^{\prime}\right) \\
E_{C}=2\left(\left(t-t^{\prime}\right)\left(x-v t^{\prime}-x_{0}\right) \operatorname{tg} \gamma+\left(t_{0}+t-t^{\prime}\right) z\right) \\
\xi=x-v\left(t+t_{0}\right)-x_{0} \\
t_{c}=l / v \\
t^{\prime \prime}=t-t^{\prime}
\end{gathered}
$$

$T(x, y, z, t)$ - temperature of body $\left[\mathrm{K},{ }^{\circ} \mathrm{C}\right], l=x-x_{0}$ - length of the weld $[\mathrm{m}], x_{0}, y_{0}-$ coordinates of weld beginning $[\mathrm{m}], \dot{q}$ - power of heat source [W], $v$ - velocity of heat source $[\mathrm{m} / \mathrm{s}], \gamma$ [degrees] - tilt angle of heat source (electrode) denotes the angle between the source axis and the perpendicular to the surface of the plate.

\subsubsection{Example of calculations}

Calculations of the temperature field have been conducted for a GMAW surfaced S235 steel plate of length $0.6 \mathrm{~m}$, width $0.4 \mathrm{~m}$ and thickness $0.03 \mathrm{~m}$. Numerical simulations were performed with the author's program created in Borland Delphi environment using Borland Pascal language. Thermal properties of surfaced material are determined by $a=8 \cdot 10^{-6} \mathrm{~m}^{2} / \mathrm{s}, c=670 \mathrm{~J} /(\mathrm{kg} \mathrm{K})$ and $\rho=7800 \mathrm{~kg} / \mathrm{m}^{3}$, $(c \rho=5.2 \mathrm{MJ} /(\mathrm{m} \mathrm{K})$. It has been assumed in calculations that the heat source of power $5488 \mathrm{~W}$ with Gaussian distribution of power density determined by $z_{0}=0.007 \mathrm{~m}$ and $t_{0}=0.5 \mathrm{~s}$. Power of heat source corresponds to the parameters of surfacing using in experiment: voltage $U=28 \mathrm{~V}$, current $I=280 \mathrm{~A}$ and arc efficiency $\eta=0.7$ according to the formula:

$$
\dot{q}=\eta U I
$$

Numerical simulations have been conducted for a heat source moving across the plate surface along the axis of symmetry with velocity $v=0.007 \mathrm{~m} / \mathrm{s}$ with initial and terminal coordinates of motion $x_{0}=0.2 \mathrm{~m}$ and $x_{k}=0.4 \mathrm{~m}$ respectively and for heat source tilt angles $\gamma-30^{\circ}, 0$ and $30^{\circ}$ respectively. Calculations of temperature have been conducted in the whole element. Due to significant temperature gradients in the heat affected zone, figures presented below contain only selected fragments of cross sections and surface, sufficient to reveal the difference in distributions. The temperature field in longitudinal sections of elements (in the axis of heat trajectory, $y=0$ ) for time $t=21 \mathrm{~s}$ (heat source is located at $x=0.34 \mathrm{~m}$ ) is presented in Figures 17-19. The tilt of heat source results in a decrease in its deposition depth. In the case of tilt opposite to the sense of the velocity vector of the heat 
source, maximum temperature values are much higher than in the case of contrary or perpendicular tilt, while the head of isotherms is shifted towards the motion of the heat source.

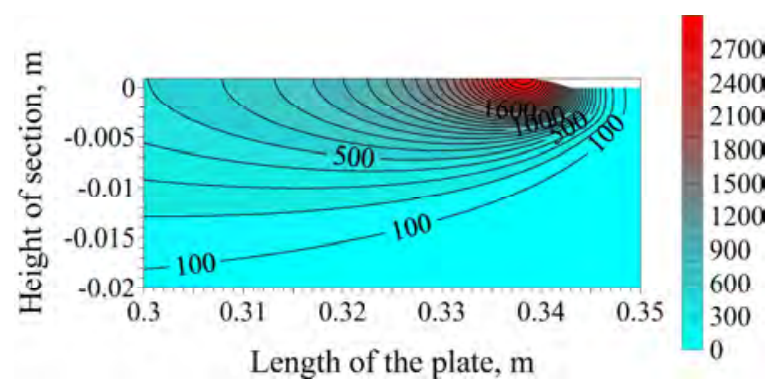

Fig. 17. Temperature distributions in longitudinal sections of plates $(y=0)$ for time $t=21 \mathrm{~s}$ and $\gamma=-30^{\circ}$

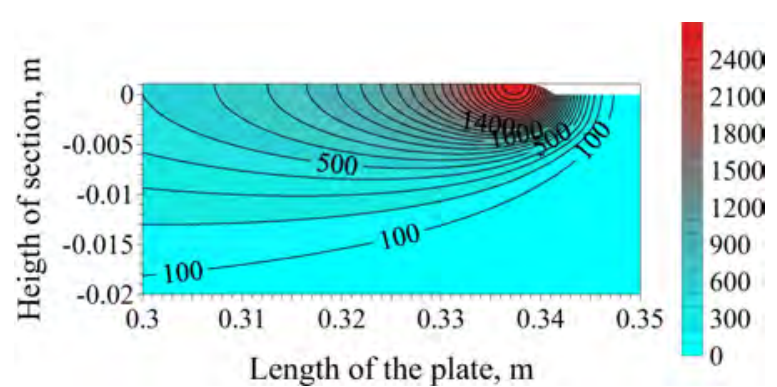

Fig. 18. Temperature distributions in longitudinal sections of plates $(y=0)$ for time $t=21 \mathrm{~s}$ and $\gamma=0$

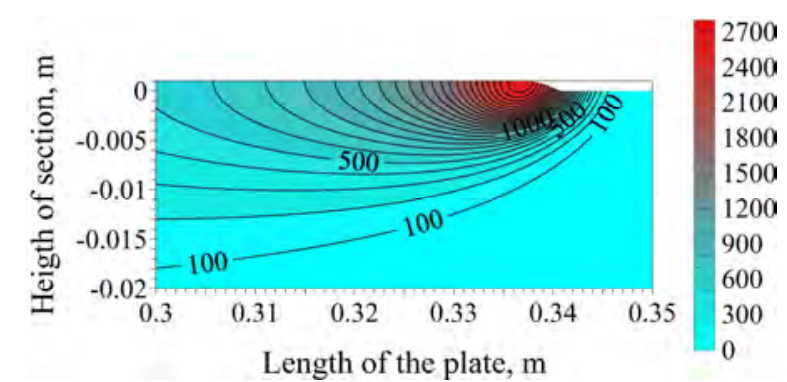

Fig. 19. Temperature distributions in longitudinal sections of plates $(y=0)$ for time $t=21 \mathrm{~s}$ and $\gamma=30^{\circ}$

Maximum temperature values in this section are presented in Figures 20-22. At point $z=0, y=0$ these values for angles $\gamma=-30^{\circ}, 0$ and $30^{\circ}$ are equal to 2864, 2574.4 and $2683.6^{\circ} \mathrm{C}$ respectively. The tilt of the heat source causes a decrease in heating depth of the material to a certain temperature (e.g. $\left.500^{\circ} \mathrm{C}\right)$ when the maximum temperature on the surface of the plate is increased. If the axis source is tilted opposite to the sense of velocity vector of the heat source, maximum 
temperature is higher than in the case of contrary tilt and much higher than for the heat source perpendicular to the plate.

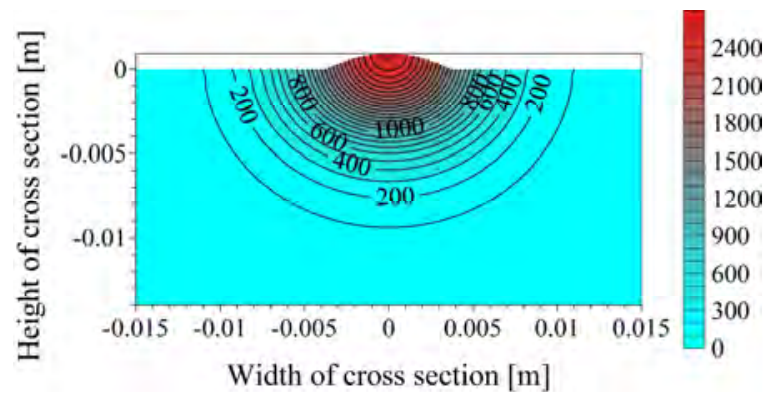

Fig. 20. Isotherms of maximum temperature in cross sections of the plate $(x=0.34 \mathrm{~m})$ : $\gamma=-30^{\circ}$

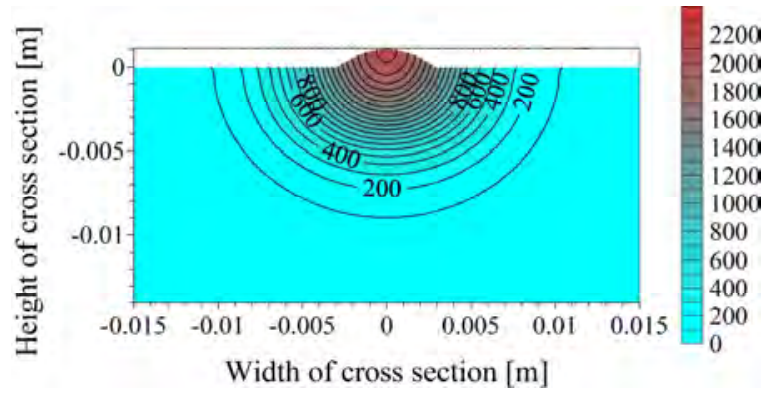

Fig. 21. Isotherms of maximum temperature in cross sections of the plate $(x=0.34 \mathrm{~m})$ : $\gamma=0$

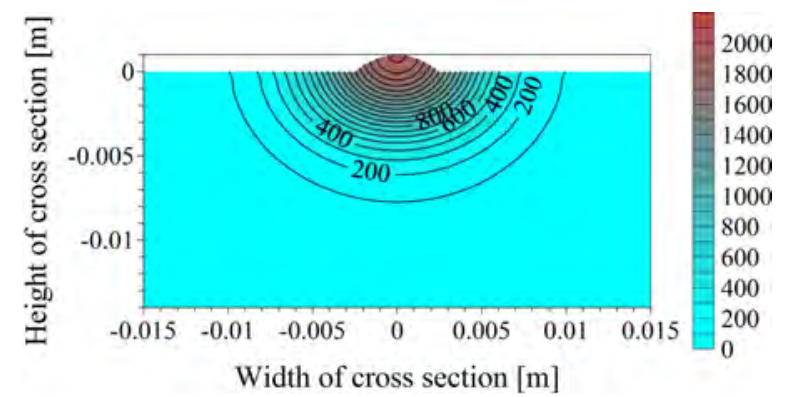

Fig. 22. Isotherms of maximum temperature in cross sections of the plate $(x=0.34 \mathrm{~m})$ :

$$
\gamma=30^{\circ}
$$

Thermal cycles at point with coordinates $x=0.34, y=0, z=0$ (surface of the plate) illustrates Figure 23. Peaks of temperature for particular angles of heat source tilt are shifted in relation to each other in time and they have different values. Table 1 shows the temperature values for selected times - the asterisk indicates the maximum temperature values in particular cycles. At time $21.25 \mathrm{~s}$, the temperature 
during weld surfacing with the angle $\gamma=-30^{\circ}$ is highest and $288.1^{\circ} \mathrm{C}$ higher than that of the angle $\gamma=0$ and $291.1^{\circ} \mathrm{C}$ higher than at the angle $\gamma=30^{\circ}$.

Table 1

Temperatures at point $x=0.34, y=0, z=0$ for selected times

\begin{tabular}{|c|c|c|c|}
\hline \multirow{2}{*}{$\begin{array}{c}\text { Time } \\
{[\mathrm{s}]}\end{array}$} & \multicolumn{3}{|c|}{$\begin{array}{c}\text { Temperature } \\
{\left[{ }^{\circ} \mathrm{C}\right]}\end{array}$} \\
\cline { 2 - 4 } & $\gamma=-30^{\circ}$ & $\gamma=0^{\circ}$ & $\gamma=30^{\circ}$ \\
\hline 21.25 & $2730.9^{*}$ & 2442.8 & 2439.8 \\
\hline 21.40 & 2683.4 & $2500.1^{*}$ & 2590.1 \\
\hline 21.50 & 2599.7 & 2477.7 & $2619.9^{*}$ \\
\hline
\end{tabular}

As the heat source moves, the temperature at the point under consideration decreases with $\gamma=-30^{\circ}$, while in the other two cases it increases to the maximum. At time $21.5 \mathrm{~s}$ with the angle $\gamma=30^{\circ}$ it reaches a maximum value that is $20.2^{\circ} \mathrm{C}$ and $142.2^{\circ} \mathrm{C}$ higher than at the same time during surfacing with angles $\gamma=-30^{\circ}$ and $\gamma=0$, respectively.

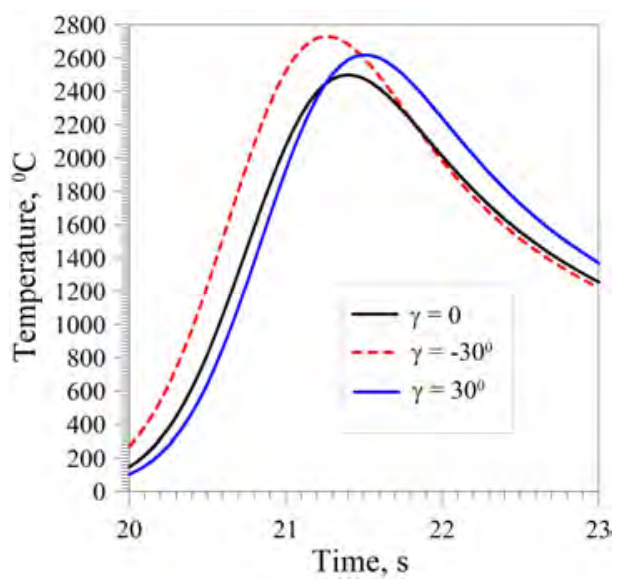

Fig. 23. Thermal cycles at points with coordinates $x=0.34 \mathrm{~m}, y=0, z=0$

\section{Summary}

Modeling of the weld surfacing and build up processes involves the appropriate determination of the temperature field, the quantitative evaluation of the phase transformations and the determination of the strains and temporary stresses (occurring in the surfacing process) and the residual stresses after the surfacing. A origin for the accepted modeling of the temperature field therefore is the adoption of a suitable heat source, the restoration of the corresponding electrode movement and its position relative to the surfaced system. 
The suggested analytical solution enables one to determine temperature at any place and time of the built up of weld surfaced object. The mathematical model takes into consideration heating by a moving thermal welding zigzag, as well as self-cooling of already heated areas. Accepted parameters of weave bead building up enable one to obtain weld beads around 26-28 mm wide, weld reinforcement $4 \mathrm{~mm}$ high and fusion $2 \mathrm{~mm}$ proved experimentally [7].

The analysis of the results of the temperature field calculations for different angles of electrode inclination with respect to its motion direction allowed us to formulate the following conclusions:

- the electrode inclination reduces the depth of material heating In the case of the tilt angle of the electrode $\gamma=-30^{\circ}$ (opposite the velocity vector) obtained heat affected zone is broader, but shallower, and temperatures are significantly higher than for angle $\gamma \geq 0$. For $\gamma=30^{\circ}$ the dimensions of the heated zone are indirect, while the narrowest and deepest heated zone are obtained for the angle $\gamma \geq 0$;

- when the source is tilted opposite, the maximum temperature value is significantly higher than the inclined source in the direction of motion or perpendicular to the plate surface, and the isotherm face is shifted more toward the source movement than it is the other two cases.

In addition, when the source is tilted opposite, the maximum temperature is significantly higher than the inclined source in the direction of movement or perpendicular to the surface of the object. The electrode tilt causes not only changes in heating depth, but varied temperature distributions, including distinctly different heat cycles in the same geometric points, may imply significant differences in phase transformation, strain and stress fields.

\section{References}

[1] Ferenc K., Welding, WNT, Warszawa 2007 (in Polish).

[2] Rosenthal D., Mathematical theory of heat distribution during welding and cutting. Welding J. 1941, 20, 220s-234s.

[3] Rykalin N., Fundamentals of Heat Flow in Welding. AN SSSR, Moskva 1947.

[4] Carslaw H.S., Jaeger J.C., Conduction of Heat in Solids, Clarendon Press, London 1996.

[5] Eagar T.W., Tsai N.S., Temperature fields produced by traveling distributed heat sources, Welding J. 1983, 62, 346-355.

[6] Goldak J., Chakravarti A., Bibby M., A new finite element model for welding heat sources, Metal. Trans. 1984, 15B, 299-305.

[7] Hongyuan F., Qingguo M., Wenli X., Shude J., New general double ellipsoid heat source model, Science and Technology Welding and Joining 2005, 10, 361-368.

[8] Winczek J., Analytical solution to transient temperature field in a half-infinite body caused by moving volumetric heat source, Int. J. Heat Mass Transf. 2010, 53, 5774-5781. 
[9] Parkitny R., Winczek J., Analytical solution of temporary temperature field in half-infinite body caused by moving tilted volumetric heat source, Int. J. Heat Mass Transf. 2013, 60, 469-479.

[10] Vishnu P.R., Li W.B., Easterling K.E., Heat-flow model for pulsed welding, Mater. Sci. Techn. 1991, 7, 649-659.

[11] Mathematical Modelling of Weld Phenomena, eds. H. Cerjak, K.E. Easterling, The Institute of Materials, London 1993. 